Original research article

\title{
Gut microbial dysbiosis and its association with esophageal cancer
}

\author{
Hafiz Muhammad Ishaq ${ }^{1,2}$, Imran Shair Mohammad ${ }^{3}$, Kiran Sher Muhammad ${ }^{4}$, Huan Li ${ }^{5}$, \\ Rao Zahid Abbas ${ }^{6}$, Zia ud Din Sindhu ${ }^{6}$, Shakir Ullah ${ }^{1}$, Yang Fan ${ }^{7}$, Abbas Sadiq ${ }^{8}$, \\ Muhammad Asif Raza ${ }^{2}$, Riaz Hussain ${ }^{9}$, Hafiz Muhammad Arshad ${ }^{10}$, Iahtasham Khan ${ }^{11}$, \\ Muhammad Umair Waqas ${ }^{2}$, Aziz Ul-Rahman ${ }^{2}$, Riffat Yasin ${ }^{2}$, Atif Rehman ${ }^{2}$, Rana Waseem Akhtar ${ }^{2}$, \\ Jiru $\mathrm{Xu}^{1 *}$
}

1 Xi'an Jiaotong University, School of Medicine, Key Laboratory of Environment and Genes Related to Diseases of Chinese Ministry of Education, Department of Microbiology and Immunology, Xi'an, China

2 Muhammad Nawaz Shareef University of Agriculture Multan, Department of Veterinary and Animal Sciences, Multan, Pakistan

3 Sun Yat-sen University, University Town, School of Pharmaceutical Sciences, Guangzhou 510006, China

4 University of Agriculture, Department of Zoology Wild-life and Fisheries, Faisalabad, Pakistan

5 Xi'an Mental Health Centre, Xi'an, China

6 University of Agriculture Faisalabad-38040, Department of Parasitology, Faisalabad, Pakistan

7 Xinxiang Medical University, School of Basic Medical Science, Department of Microbiology, Xinxiang, China

8 Faculty of Veterinary and Animal Sciences Lahore, Department of Pathology, Lahore, Pakistan

9 Islamia University of Bahawalpur-63100, Faculty of Veterinary and Animal Sciences, Department of Pathology, Bahawalpur, Pakistan

${ }^{10}$ Bahauddin Zakariya University, Department of Clinical Sciences, Multan, Pakistan

${ }^{11}$ University of Veterinary and Animal Sciences, Department of Clinical Sciences, Section of Epidemiology and public Health, Lahore Sub-campus Jhang, Pakistan

\begin{abstract}
Due to its aggressive nature and low survival rate, esophageal cancer is one of the deadliest cancer. While the intestinal microbiome significantly influences human health and disease. This research aimed to investigate and characterize the relative abundance of intestinal bacterial composition in esophageal cancer patients. The fecal samples were collected from esophageal cancer patients $(n=15)$ and healthy volunteers $(n=10)$. The PCR-DGGE was carried out by focusing on the V3 region of the $16 S$ rRNA gene, and qPCR was performed for Bacteroides vulgatus, Escherichia coli, Bifidobacterium, Clostridium leptum and Lactobacillus. High-throughput sequencing of the $16 \mathrm{~S}$ rRNA gene targeting the V3+V4 region was performed on 20 randomly selected samples. PCR-DGGE and High-throughput diversity results showed a significant alteration of gut bacterial composition between the experimental and control groups, which indicates the gut microbial dysbiosis in esophageal cancer patients. At the phylum level, there was significant enrichment of Bacteroidetes, while a non-significant decrease of Firmicutes in the experimental group. At family statistics, a significantly higher level of Bacteroidaceae and Enterobacteriaceae, while a significantly lower abundance of Prevotellaceae and Veillonellaceae were observed. There was a significantly high prevalence of genera Bacteroides, Escherichia-Shigella, while a significantly lower abundance of Prevotella_9 and Dialister in the experimental group as compared to the control group. Furthermore, the species analysis also showed significantly raised level of Bacteroides vulgatus and Escherichia coli in the experimental group. These findings revealed a significant gut microbial dysbiosis in esophageal cancer patients. So, the current study can be used for the understanding of esophageal cancer treatment, disease pathway, mechanism, and probiotic development.
\end{abstract}

Keywords: DGGE; Esophageal cancer; High-throughput sequencing; Intestinal dysbiosis; Microbiome

\section{Highlights:}

- The gut microbial dysbiosis was observed in esophageal cancer patients

- A significant enrichment of Bacteroidetes, but non-significant decrease in Firmicutes was observed in esophageal cancer patients.

- Family statistics showed significant high levels of Bacteroidaceae and Enterobacteriaceae in esophageal cancer patients.

- The high abundant genera: Bacteroides, Escherichia-Shigella; lower abundance genera: Prevotella_9 and Dialister.

- The species analysis found a significant increase of Bacteroides vulgatus and Escherichia coli in esophageal cancer patients.

\footnotetext{
* Corresponding author: Jiru Xu, Xi'an Jiaotong University, School of Medicine, Key Laboratory of Environment and Genes Related to Diseases of Chinese Ministry of Education, Department of Microbiology and Immunology, Xi'an, China; e-mail: xujiru@xjtu.edu.cn http://doi.org/10.32725/jab.2021.005
}

Submitted: 2020-06-08 • Accepted: 2021-01-22 • Prepublished online: 2021-02-03

J Appl Biomed 19/1: 1-13 • EISSN 1214-0287 • ISSN 1214-021X

(c) 2021 The Authors. Published by University of South Bohemia in České Budějovice, Faculty of Health and Social Sciences.

This is an open access article under the CC BY-NC-ND license. 


\section{Introduction}

Human intestinal flora is a significant health determinant, as the microorganisms perform metabolic, trophic, and protective functions to protect the body from many diseases (Guarner and Malagelada, 2003). Different factors such as age, diet, and disease could affect their composition and function (Power et al., 2013). An average individual contains approximately a hundred trillion gut microorganisms, important in host homeostasis, i.e., immunity, nutrition, metabolism and absorption (Walsh et al., 2014). Alteration in the gut microbial composition can cause various diseases such as type II diabetes, viral diarrhea, Crohn's disease, colitis, inflammatory bowel disease, Grave's disease, smoking, and asthma (Ishaq et al., 2017b; 2018a, b). Cancer is a leading cause of death worldwide (Hameed et al., 2017; Mohammad et al., 2018a, b; 2020a, b). Malignancy like esophageal cancer is one of the lethal and aggressive disorders, increased dramatically in the last few decades. Due to the lack of early signs and symptoms, most esophageal cancer cases are diagnosed in its advanced stages; therefore, it only hits a survival rate of 5 years (Huang and $\mathrm{Yu}, 2018$ ). It can be divided into two predominant histological categories (i) squamous cell carcinoma and (ii) adenocarcinoma (Triboulet and Mariette, 2008). The adenocarcinoma has been observed in the distal part of the esophagus that dominates in the Western part of the globe. However, squamous cell cancer grows in the middle part of the thoracic esophagus and prevails in the Eastern part of the world. Squamous cell carcinoma and adenocarcinoma can be differentiated on the basis of molecular characteristics such as squamous cell carcinomas frequently exhibited genomic augmentations of SOX2, CCND1, or TP63, while VEGFA, ERBB2, GATA6 and GATA4 commonly amplified in adenocarcinoma (The Cancer Genome Atlas Research Network, 2017). Therefore, multimodal therapeutics strategies, including chemotherapy and surgery have been employed to treat this, but the prognosis is still very poor (Baba et al., 2014). However, the gut bacteria compete for nutrients and produce the bacteriocins, protecting the intestine lining from colonization (Guarner and Malagelada, 2003). In line with this, alteration in gut microbial composition could lead to various types of cancers including intestine, stomach, breast or lung. To date, the link between intestinal bacteria and esophageal cancer has not been explored. Thus, current study was designed to elucidate the overall composition along with variation in intestinal bacteria in esophageal cancer patients. PCR-DGGE, qPCR and highly sensitive metagenomics Illumina based HIseq2500 High-throughput sequencing was used to monitor the gut microbial diversities and similarities in esophageal cancer patients in comparison with healthy control. The current study proves a significant disparity of bacterial community richness between the two groups, with certain intestinal microbes illustrating significantly lower or higher abundance, as illustrated in Fig. 1.

\section{Material and methods}

\section{Ethical statement}

The study protocol was approved by the School of Medicine, Xi'an Jiaotong University Ethics Committee. Furthermore, all procedures performed in this study were by the laws and rules of the 1964 Helsinki declaration and its later amendments or comparable ethical standards.

Informed consent: Written informed consent was obtained from all individual participants included in the study.

\section{Collection of fecal samples}

A total of 15 stool samples were collected from both squamous cell carcinoma and adenocarcinoma esophageal cancer patients (10 males and 5 females, 45-60 year age) and 10 from healthy individuals ( 6 males and 4 females, $45-60$ years age) by using
FINDINGS

Phylum

Bacteroidetes, Firmicutes

Family

Bacteroidaceae, Enterobacteriaceae

Prevotellaceae, Veillonellaceae

Genera

Bacteroides, Escherichia-Shigella,

Prevotella_9, Dialister

Species

Bacteroides vulgatus and

Escherichia coli

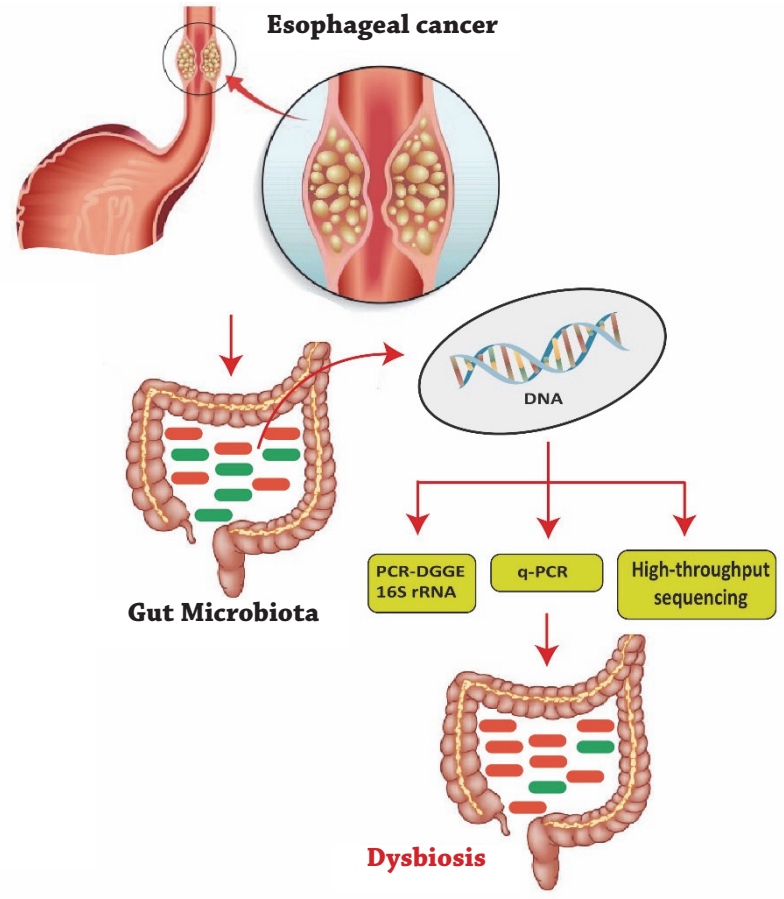

Fig. 1. Gut microbial dysbiosis in esophageal cancer patients 
a sterile cup. The type and grade of esophageal cancer patients are described in Suppl. Table 1. Each participant's questionnaire was filled containing information on gender, health, age, dietary habits, and body weight. Collected stool samples were kept in an icebox within $4 \mathrm{~h}$ of fecal reflex. The fecal samples were stored at $-80{ }^{\circ} \mathrm{C}$ in the lab until DNA extraction. The esophageal cancer patients and healthy subjects had no history of antibiotics, prebiotics, and probiotics intake 30 days before sample collection.

\section{Bacterial DNA extraction by using fecal samples}

QIAGEN (Germany, Hilden) stool kit was used for DNA extraction after thawing of stool sample with the initial bead-beating step (5000 rpm about $30 \mathrm{~s}$ ). The bacterial DNA concentration was assessed by using Nano Photometer (Germany IMPLEN).

\section{PCR augmentation for DGGE}

PCR-DGGE was used to investigate the intestinal bacterial composition fingerprinting. For this purpose, the $\mathrm{V} 3$ region of the 16S rRNA gene of extracted DNA was amplified by using universal linkage primers (Suppl. Table 2). $50 \mu \mathrm{l}$ PCR reaction mixture contained $200 \mathrm{mM}$ of deoxynucleotide triphosphate (dNTP), $20 \mathrm{pmol}$ of two primers, $\mathrm{MgCl}_{2} 2.5 \mathrm{mM}, 10 \times$ buffer $2.5 \mathrm{mM}$, Taq $2 \mathrm{U}$ of (Promega) DNA polymerase, and $2 \mu \mathrm{l}$ (approximately $120 \mathrm{ng}$ ) stool bacterial DNA. PCR Amplification was carried out in thermocycler (USA ABI2720) through touchdown PCR programming. Agarose gel was used for the PCR product's electrophoresis and dipped in Ethidium bromide solution to visualize under UV light.

\section{Denature gradient gel electrophoresis}

Universal Mutation Detection System (Bio-Rad, USA) DCodeTM was used for the denaturing gradient gel electrophoresis experiment. Amplified PCR product of total bacteria of each stool sample was loaded in (w/v) 8\% (37.5: 1 acrylamide-bis,) DGGE polyacrylamide gel inside $1 \times$ TAE buffer tank, containing linear denaturing grade 30 65\%. The DGGE gel was electrophoresed at $90 \mathrm{~V}$ for $14 \mathrm{~h}$ at a constant temperature of $60{ }^{\circ} \mathrm{C}$. The bacterial diversity of each sample was calculated by using the relative band intensity of DGGE profiles and total numbers of bands. Syngene (USA) software was used to calculate the similarity index through Dice's similarity coefficient. Unweighted pair UPGMA (arithmetic averages) was used to establish a dendrogram.

\section{Statistical analysis of DGGE band pattern}

Bacterial diversity in DGGE profiles was assessed through the Shannon-Weaver index of diversity $\left(H^{1}\right)$. Similarity index and DGGE profile's cluster analysis were done through the UPGMA method (band-based Dice similarity coefficient). Algorithm cluster analysis with (UPGMA) and arithmetic averages were used in the unweighted group pair method to estimate dendrograms.

$$
\begin{aligned}
& \text { Shannon-Weaver diversity index }\left(H^{1}\right) \text { was calculated as, } \\
& \text { Shannon-Weaver diversity index }\left(H^{1}\right)={ }_{-} \sum_{i=1}^{S}(P i)(\operatorname{In} P i)
\end{aligned}
$$

\section{Gel bands excision and sequencing}

A sterilized scalpel blade was used to cut the prominent bands of the DGGE gel profile. The excised gel bands were collected in a $1.5 \mathrm{ml}$ tube; approximately $50 \mathrm{ml}$ sterilized water was added and placed for $30 \mathrm{~min}$ at $37^{\circ} \mathrm{C}$. After centrifugation, the V3 region 16S rRNA gene was amplified by adding $8 \mu \mathrm{l}$ DNA water (as a template) by using the same primers but without
GC-clamps. The PCR re-amplified product was evaluated by using $\mathrm{ABI} 3500 \mathrm{xL}$ for sequencing. Sequences were analyzed by applying BLAST database software to identify the genus and species.

\section{qPCR analysis}

Bio-Rad (USA) CFX96 was used to perform the qPCR quantification. Total $20 \mu \mathrm{l}$ PCR reaction mixture contains $10 \mu \mathrm{l}$ of $2 \times$ SYBR Green (TOYOBO, Japan), $1 \mu$ l of both primers (Suppl. Table 2), $2 \mu \mathrm{l}$ fecal DNA, and $6 \mu \mathrm{l} \mathrm{H} \mathrm{H}_{2} \mathrm{O}$. Lactobacillus (NWS1), Bifidobacterium (CICC 6186), Clostridium leptum (YIT 6169), Escherichia coli (NWS2), and Bacteroides vulgatus (CICC 22938) were used as standard strains. Data Statistics were, the approximate average logarithm of fecal bacterial DNA genetic amplicon, copy numbers present in $1 \mathrm{~g}$ fecal mass.

\section{Illumina based HiSeq 2500 High-throughput sequencing}

Illumina-based high-throughput was applied to 20 randomly selected fecal samples for metagenomic analysis (10 patients' samples and 10 samples as control from healthy volunteers). According to the previous methodology, the $16 \mathrm{~S}$ rRNA gene with $\mathrm{V} 3+\mathrm{V} 4$ location was amplified by using a primer: F-515-(GTGCCAGCMGCCGCGGTAA), R-806- (GGACTACHVGGGTWTCTAAT) to manufacture the amplicon libraries. The paired-end High-throughput sequencing was performed on Illumina Hiseq 2500 platform using the manufacturer's standard procedure. Software packages FLASH and QIIME were used to screen and assembled the raw data. The UCLUST was computed to cluster OTUs sequences (Operational Taxonomic Units) with a 97\% threshold. Also, a Classifier (RDP) was used to allot taxa level OTU. QIIME was used to study the diversities like Chao1, Good's coverage Simpson, Shannon, and ACE. The OTU cluster was manufactured by using the QIIME pipeline, which was then imported in MEGAN-4 and matched with the taxonomic database of NCBI, and fluctuations of the intestinal bacterial community were calculated.

\section{Statistics analysis}

Statistical analysis was performed in Microsoft Excel 2010 and statistic software GraphPad prism 7. A nonparametric unpaired $t$-test was computed, and significance value was measured as $(P<0.05)$.

\section{Results}

\section{DGGE profiles analysis}

Analytical and experimental process of denaturing gradient gel electrophoresis was performed with amplified PCR product by using the universal primers of the V3 region of the 16S rRNA gene in both esophageal cancer patients and healthy control groups. In Fig. 2A, (D1-D8) shows the esophageal cancer patients samples while (C1-C5) represents the healthy controls. Similarly, in Fig. 2C, (D9-D15) shows esophageal cancer patients' samples and (C6-C10) to healthy controls. A total of 194 bands were detected by using Syngene software (Van Der Gucht et al., 2001) in 15 tracks of esophageal cancer patients with a band average $(12.12 \pm 3.37)$. A sum of 71 bands was identified in 10 tracks of healthy volunteers group with an average of $(7.73 \pm 1.60)$, showed there was a significant band difference $(P<0.0007)$ between esophageal cancer and healthy control. The increased band numbers indicate the bacterial overgrowth in the esophageal cancer patients group. To inves- 
tigate the intestinal microbial diversity between esophageal cancer patients and healthy control, the Shannon Weaver diversity index $\left(H^{1}\right)$ was calculated. Diversity results illustrated (3.06 \pm 0.69 vs. $2.52 \pm 0.26)$ in a significant $(P<0.017)$ disparity between diseased and control groups also indicates a significant bacterial overgrowth in esophageal cancer patients as compared to healthy controls. Dice similarity coefficient and UPGMA dendrogram of the entire DGGE profiles were evaluated using Syngene software shown in Fig. 2B and Fig. 2D. Dice similarity coefficient based on band intensity of DGGE profile of esophageal cancer patients and control group, the mean similarity index comparison between two groups was $(0.340 \pm 0.164)$ and $(0.271 \pm 0.147)$, respectively (Suppl. Table 3 ). The mean similarity index between the two groups was $(0.174 \pm 0.155)$, indicating the lesser value in inter-group than intra-group, signifying that esophageal cancer patients' gut microbial composition was different from healthy individuals.

\section{Dominant band analysis with sequencing results}

A total of 23 gel bands were excised from two DGGE gel profiles. From Fig. 2A, 13 prominent bands were excised from the DGGE profile for quantity analysis. Bands belonging to different tracks but at a similar position, D3b and C3a, were cut and sequenced to confirm the resolution capacity of DGGE. It was identified as Prevotella copri with 96\% similarity. Similarly, from Fig. 2C, 10 bands were cut. Also, to check the resolution ability of DGGE gel, bands D13a band D14a were cut and sequenced and identified Klebsiella pneumonia with 93\% similarity. Taxonomic reorganization of other bands is shown in Suppl. Table 4. BLAST software was used to analyze the sequence. Sequencing results demonstrated that the most prevalent phyla were Firmicutes, Proteobacteria, and Bacteroidetes. The two DGGE gel profiles' results indicated the prevalence of opportunistic bacteria (Escherichia coli, Bacteroides vulgatus, Bacteroides fluxus, Enterobacter sacchari, Shigella flexneri, Proteus mirabilis, Bacteroides uniformis, Bacteroides salyersiae, Bacteroides plebeius, Bacteroides oleiciplenus, Klebsiella pneumonia, Bacillus sp., Pseudomonas cremoricolorata, Enterobacter aerogenes, $\mathrm{Me}$ gasphaera sp., Bacteroides dorei) in esophageal cancer patients.

\section{qPCR quantification}

qPCR showed the copy numbers of Clostridium leptum, Bacteroides vulgatus, Lactobacillus and Bifidobacterium genera. Results demonstrated that copy number of Bifidobacterium were decreased (6.91 \pm 1.08 vs. 8.07. \pm 1.04$)$ significantly $(P<0.0068)$ in the esophageal cancer group as compared to healthy subjects, also Lactobacillus genus (7.12 \pm 1.04 vs. $7.98 \pm 0.89)$ was decreased significantly $(P<0.0221)$ in patients group. Copy
A

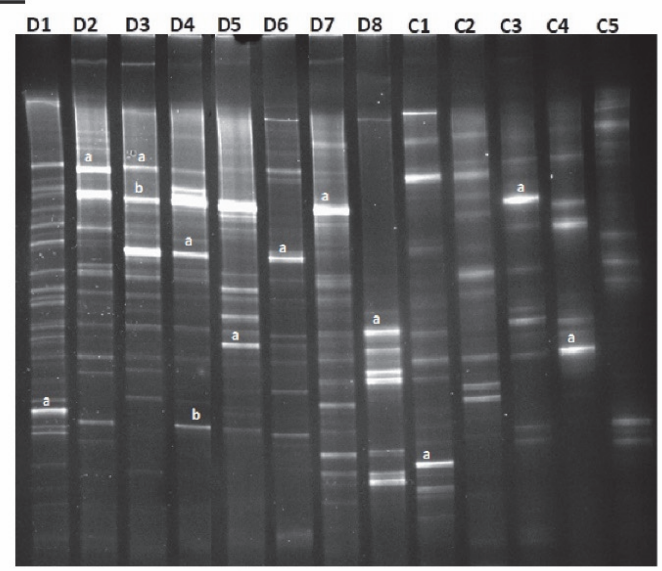

$\underline{\mathrm{C}}$

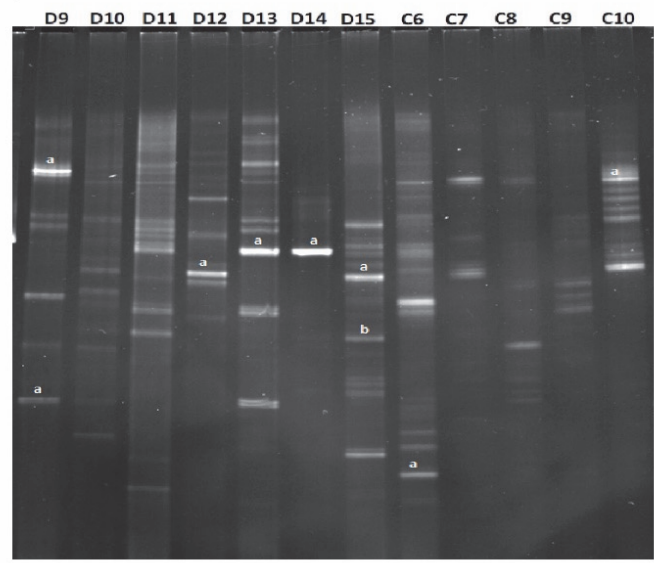

B

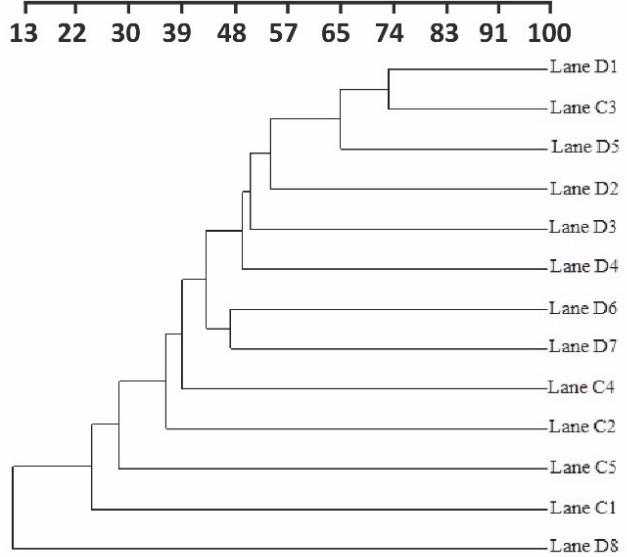

D
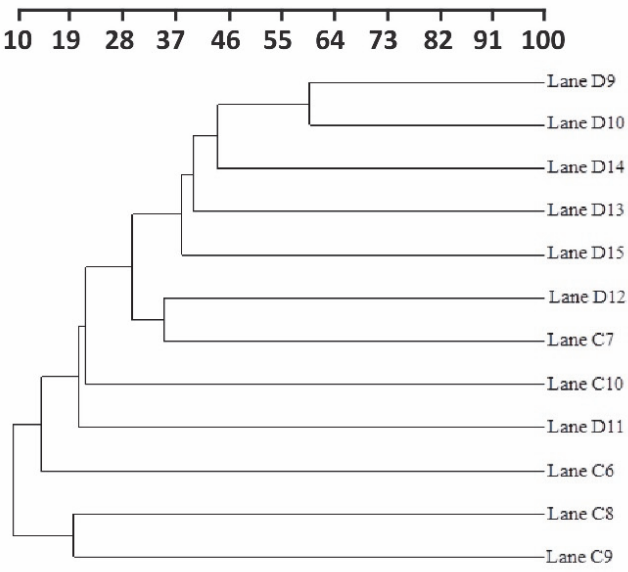

Fig. 2. (A) profiling of DGGE built between esophageal cancer (D1-D8) and controls counterparts (C1-C5). (B) Cluster estimation analysis between esophageal cancer (D1-D8) and healthy volunteers (C1-C5) groups by UPGMA application. (C) Profiling DGGE created between esophageal cancer (D9-D15) and healthy volunteers (C6-C10). (D) Cluster analysis between esophageal cancer (D9-D15) and healthy volunteers (C6-C10) groups, through UPGMA. "a" and "b" depicted in Fig. (2A) and (2C) linked with dominant bands of patients and controls. $\mathrm{D}$ and $\mathrm{C}$ represent esophageal cancer and healthy control groups, respectively. 
numbers of Bacteroides vulgatus $(7.93 \pm 1.02$ vs. $6.92 \pm 1.03)$ were increased significantly $(P<0.0119)$, also replica count of Escherichia coli $(4.59 \pm 0.78$ vs. $3.90 \pm 0.85)$ were significantly higher $(P<0.0241)$ in study group as compared to controls, while those of Clostridium leptum ( $4.39 \pm 1.18$ vs. $4.12 \pm 1.22)$ were increased non-significantly $(P<0.2928)$, Suppl. Table 5.

\section{High-throughput sequencing analysis of gene sequence} The total sequence reads were assessed about 1,883,183 at (V3 + V4 location) 16S rRNA gene of 10 esophageal cancer patients and 10 from healthy volunteers. 1,577,154 High-throughput sequencing amplicons were selected after quality assurance (control 785,941, diseased 791,213, an average/sample 78857.7) were used for further investigation. The average Taxon in all samples was 74010.35. Unique tag in the control and study group were observed $(1045.7,1324.1)$, respectively, with an average unique tag (1184.9) in all esophageal cancer samples and healthy volunteers. Total 5541 OUT was allocated in the current study (disease 2946 and control 2595, average/ sample 277.05). A total of 23698 unique tags demonstrated the whole intestinal bacterial community in this study. The average length of the High-throughput sequence was found about $418(\mathrm{bp})$ after primer deletion.

\section{Intestinal bacterial confirmation and diversification analysis}

The bacterial diversity richness was calculated at a $97 \%$ similarity level. Alpha diversity variation was estimated by Shannon, Evenness ACE, Simpson, Observed species and Chao1 which were found significantly higher in esophageal cancer patients $(P<0.0226, P<0.0237, P<0.0348, P<0.0222$ and
$P<0.0170$, respectively) as compared to the healthy volunteers. Conversely, Good's coverage was non-significantly higher in the disease group $(P<0.0982)$. The bacterial diversity estimation in the two groups is present in Suppl. Table 6. Moreover, alpha diversity exhibits a significant bullish trend in esophageal cancer patients compared to healthy individuals, clearly reflecting intestinal bacterial overgrowth in the diseased group. Each group of bacterial DNA samples was distributed into two distant clusters (on unweighted UniFrac tree metrics) shown in Fig. 3, corresponding to the PCR-DGGE pattern.

\section{Phyla level taxa composition}

The abundance of phylum, family, genus, and species is presented with a percentage $(0.5-1 \%)$ in the current study. A total of 13 phyla were observed in High-throughput results. The significantly increased phylum was Bacteroidetes, while non-significantly decreased phyla were Firmicutes and Proteobacteria in the experimental group compared to healthy volunteers Fig. 4. Top 10 phyla statistics with percentage is shown in Suppl. Table 7, indicates the obvious dissimilarity between control and study group phyla.

\section{Family level intestinal bacteria abundance}

85 different families were found in High-throughput results; significantly increased families were Enterobacteriaceae, Bacteroidaceae, in the study group with a comparison of healthy volunteers. Whereas, the significantly lowered richness of Prevotellacea and Veillonellaceae in the study group were observed in the comparison of control, depicted in Fig. 5. The 10 most families' statistics with percentages shown in Suppl. Table 7 display differences between disease and control groups.

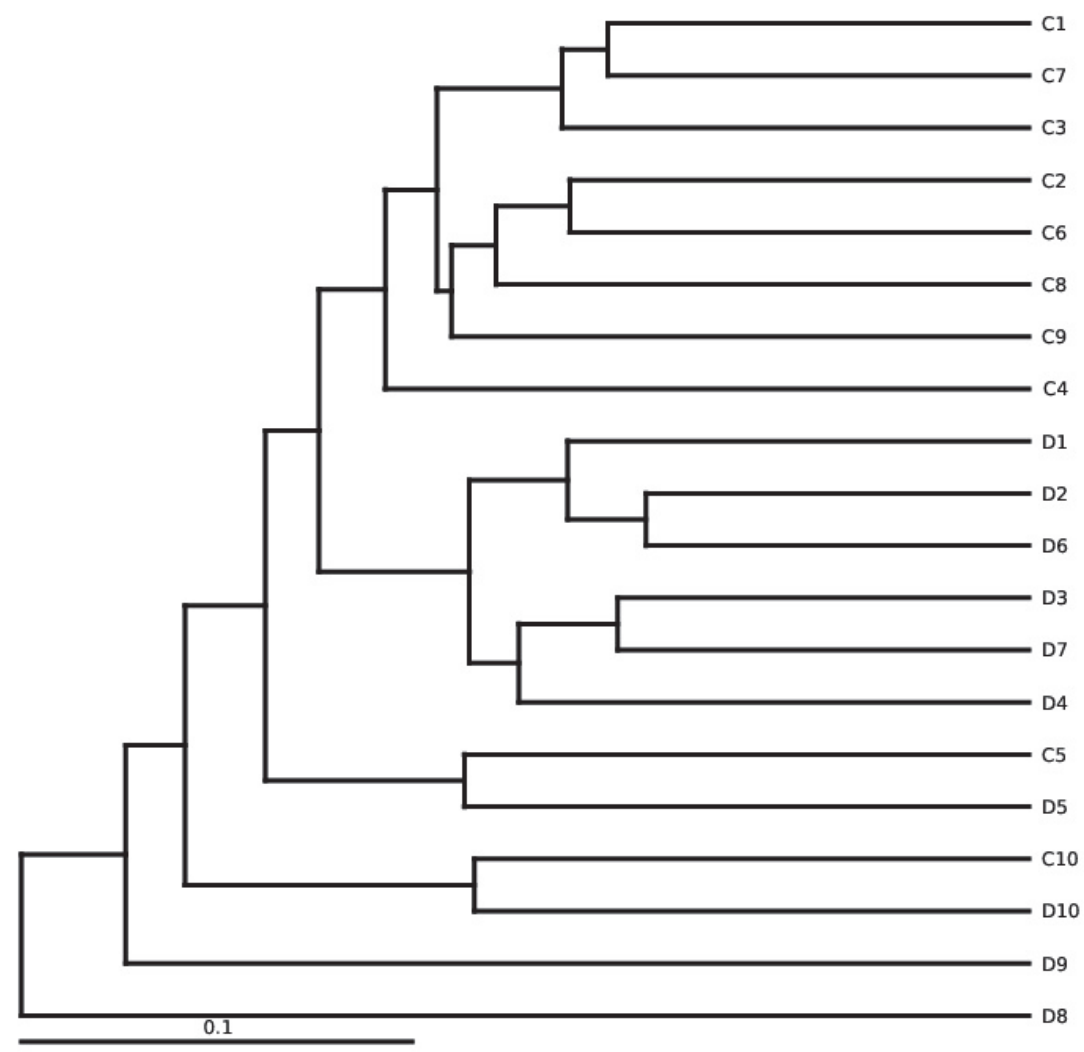

Fig. 3. High-throughput sequencing diversity analysis among esophageal cancer samples and healthy counterparts (unweight UniFrac distances based on UPGMA). D and C symbolize esophageal cancer and healthy control groups, respectively. 


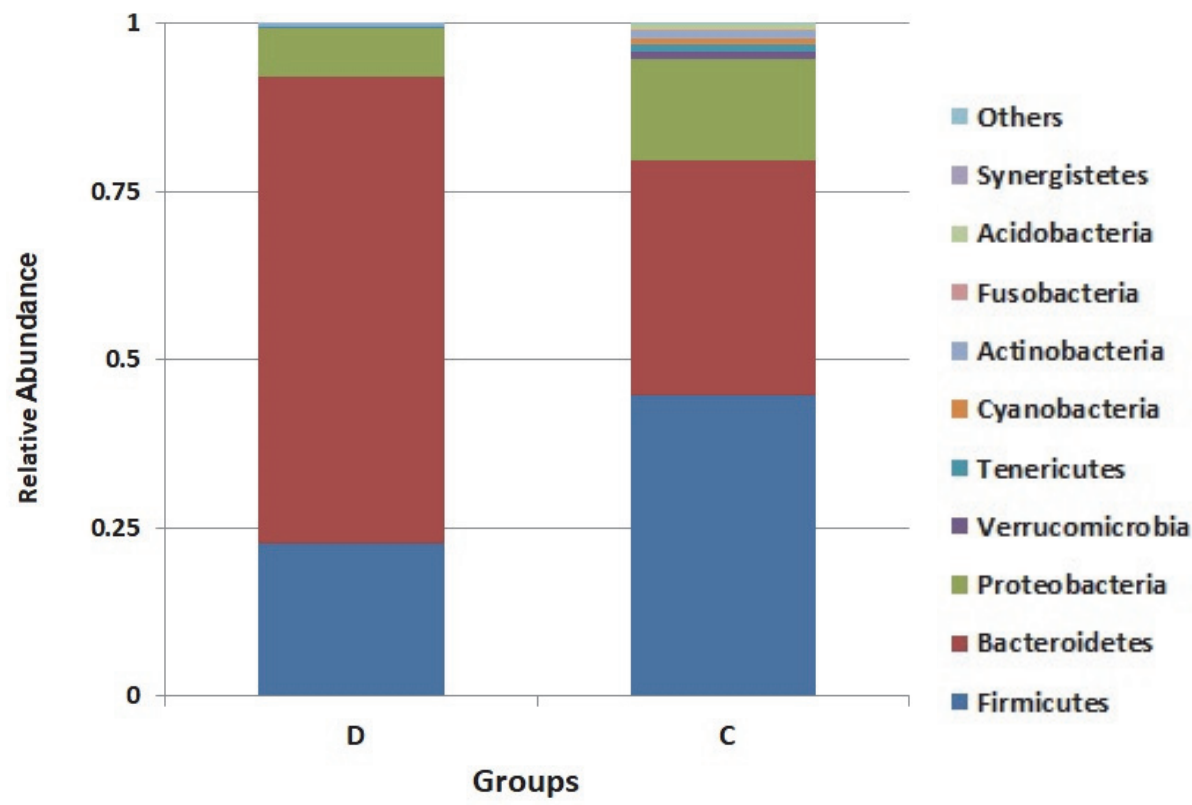

Fig. 4. Intestinal bacterial composition at phylum levels from High-throughput sequencing outcomes. The relative abundance of prevalent phyla in esophageal cancer and controls. D and C depict esophageal cancer and healthy control groups, respectively.

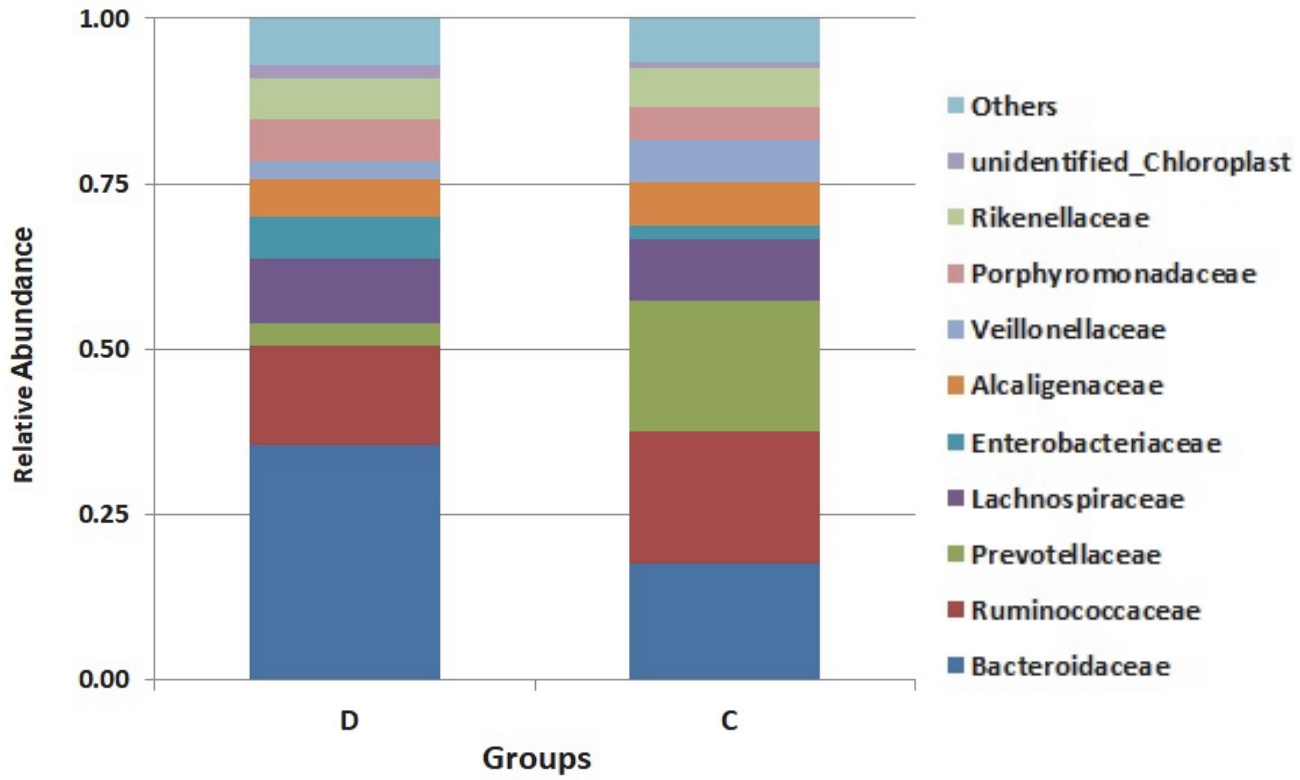

Fig. 5. Family levels High-throughput results of intestinal bacterial conformations. Dominant families' relative abundance of esophageal cancer and normal controls. D and C show esophageal cancer and control group, respectively.

\section{Genus level intestinal bacterial distribution}

189 genera were observed in High-throughput sequencing. Significantly raised levels of genera were Bacteroides, and Escherichia-Shigella in the experimental group, contrary to control. Nevertheless, significantly decreased genera in results were Prevotella_9 and Dialister, as shown in Fig. 6, Supp. Table 7. Esophageal cancer affects Phylum Bacteroidetes, family Veillonellaceae, Prevotellaceae and Enterobacteriaceae, genera
Bacteroides, Prevotella_9, Escherichia-Shigella, and Dialister. It also affects the other intestinal bacteria that may change the health status of an individual. Species results exhibited a notable difference between esophageal cancer patients and controls. Conversely, significant enrichment of Bacteroides vulgatus and Escherichia coli in esophageal cancer patients is depicted in Suppl. Table 8. 


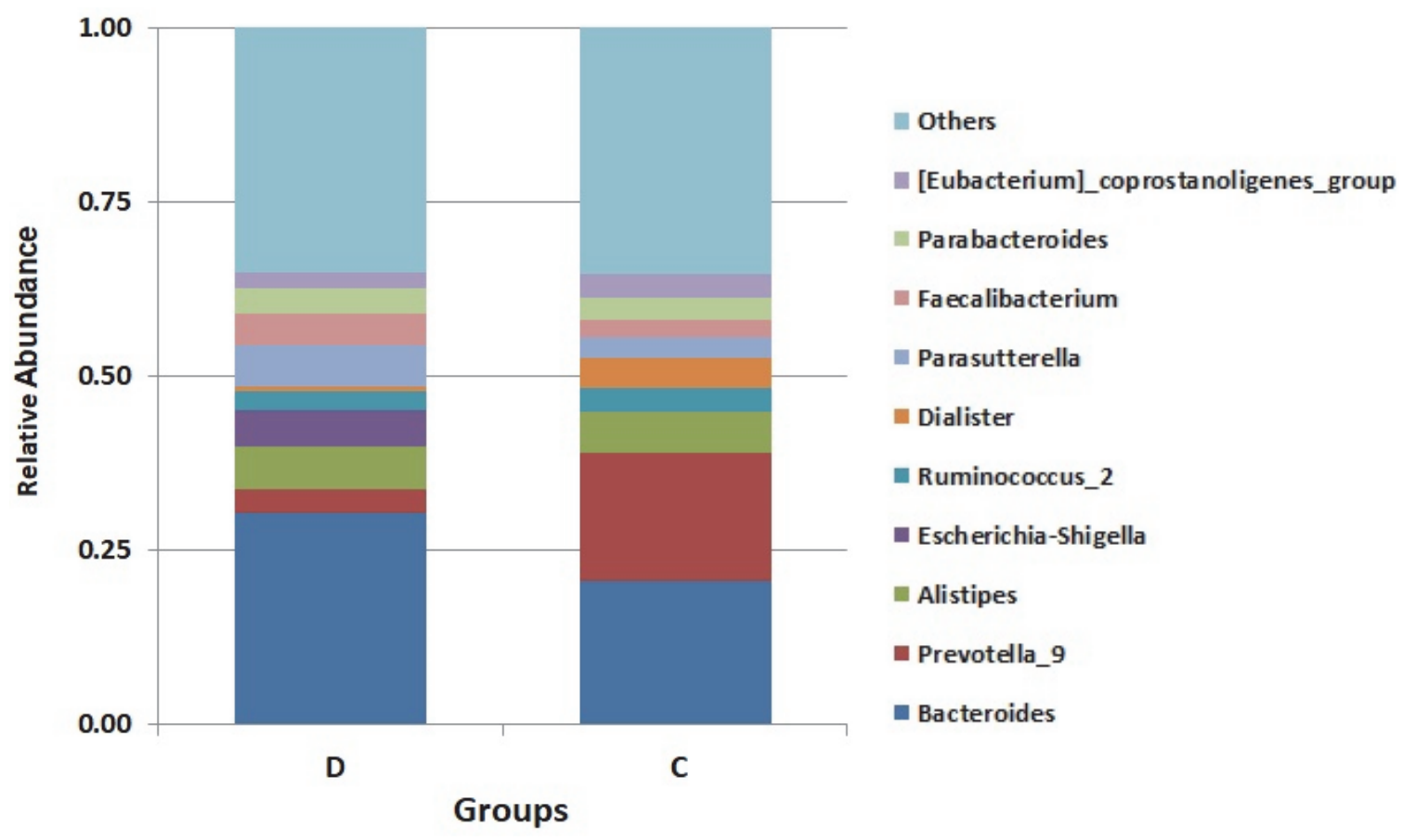

Fig. 6. Genera level intestinal bacterial compositions by High-throughput sequencing outcomes. The relative abundance of the most prevalent genera in esophageal cancer and healthy controls. D and C denote esophageal cancer and healthy control groups, respectively.

\section{Discussion}

The gut microbiome plays a vital role in the normal physiological function of the human body. There is growing evidence that gut microbial composition undergoes alteration in disease conditions, such as Crohn's disease, malnutrition, inflammatory bowel disease, colitis, obesity, and type II diabetes. On the other side, Esophageal cancer is a fast-growing tumor and it is estimated that it is the 6th leading cause of death in humans (Mao, 2016). Recently, the microbiome emerged as a critical player and driver of various types of cancer, including intestine, breast, and lung cancer.

It was hypothesized that gut flora influences the (ROS) reactive oxygen species production through immune cells. So, ROS increases the DNA damaging chance produced via oxaliplatin. This process would block the transcription and DNA replication, which results in cell death (Iida et al., 2013). In the present study, we observed a clear relationship between the gut microbiome and esophagus cancer. The results were authenticated by DGGE, qPCR, and High-throughput sequencing analysis. In $\alpha$-diversity, Shannon, Simpson, observed species Chao1, ACE, Evenness showed a significant elevation of the unique microbial population in esophageal cancer patients (Ishaq et al., 2017a).

The DGGE gut microbial similarity index statistics in esophageal cancer patient was found more abundant in intra-groups, which indicate the overgrowth of bacteria. The similarity index assessment comparison is significantly lower in the inter-group against intra-group, agreeing with previously reported results (Wu et al., 2010). Thus, the above diversity findings showed significant gut microbial dysbiosis between esophageal cancer patients and healthy volunteers. Moreover, the quantitative statistical data also showed a considerable alteration between esophageal cancer patients and control. A gut microbiome meta-analysis has been linked with irritable bowel syndrome (IBD) and obesity. It revealed that the percentage of Firmicutes and Bacteroidetes is not stable from lean to obese intestinal bacteria (Walters et al., 2014). A higher abundance of Bacteroidetes has also been linked with diseases like type II and type I diabetes and irritable bowel syndrome (Pittayanon et al., 2019). Usually, a healthy human esophagus contains a distinct microbial flora than the oral microbiome. Also, the normal esophagus possesses the Phylum Firmicutes as a significant part of the microbiota. Nevertheless, the prevalence of other phyla like Proteobacteria, Bacteroides, Actinobacteria, Fusobacteria has also been reported in previous literature (Baba et al., 2017). However, our finding showed a significant decrease and increased level of Firmicutes, Proteobacteria, and Bacteroides in gut microbiota of esophageal cancer patients compared to the healthy counterparts.

If we see the microbial families, Veillonellaceae and Prevotellaceae were significantly lesser in esophageal cancer patients than healthy volunteers aligning with current research work (Chen et al., 2016; Zhang et al., 2017). Veillonellaceae is a beneficial microbe that is closely associated with Clostridium. It helps in the production of T-regulatory immune cells (Atarashi et al., 2011). Gut microbiota plays a vital role in the immune function of the body. So, it influences the successful PDL1 (antibody) immunotherapy of cancer care patients (Garrett, 2015; Iida et al., 2013). However, a significantly higher abundance of Bacteroidaceae and Enterobacteriaceae were observed in esophageal cancer patients compared to healthy volunteers, which is aligned with preceding research of type-II diabetes individuals and intestinal flora (Wang et al., 2017).

Conversely, genera Dialister and Prevotella_9 were decreased significantly in the study group as compared to control. Lowered level Prevotella is linked with types-1 diabetes and autism and gut microbiome (Kang et al., 2013). Significantly increased genera were Bacteroides and Escherichia-Shi- 
gella, in the patient's group compared to control, agreeing with reported research of autism disorders and intestinal bacteria (Strati et al., 2017).

The current study indicates the significant reduction of Prevotella genera in the study group compared to control; Prevotella has a beneficial role in an individual's metabolism (Kovatcheva-Datchary et al., 2015). However, Prevotella is a useful commensal bacteria related to plant-based food, also linked with inflammation (Dillon et al., 2015). The results showed significantly increased values of Escherichia-Shigella in esophageal cancer patients as compared to healthy counterparts. However, Escherichia-Shigella always produces Shiga-toxin, which may cause colitis, septicemia, thrombocytopenia, GIT inflammations, urinary tract infection (Amani et al., 2015). Moreover, the raised level of Escherichia coli can also cause food-borne diseases and (UTIs) urinary tract infections (Mulvey et al., 2000). It has been documented that there is a high prevalence of Escherichia coli in esophageal carcinoma and Barrett's esophagus. Current research also showed a significantly raised level of Escherichia coli in the gut of esophageal cancer (Zaidi et al., 2016). Moreover, it has been reported TLR 1-3, 6,7 , and 9 is notably upregulated in cancer like esophageal adenocarcinoma compared to the normal healthy epithelium. It indicates the possible linkage between Escherichia coli and TLR signaling pathways. The microbes can mediate early molecular modifications in an experimental mouse model of esophageal adenocarcinoma carcinogenesis (Zaidi et al., 2016). However, a significantly raised abundance of Bacteroides vulgatus species was observed in the patient's group, which is in accordance with previous literature on viral diarrhea and gut microbiome (Ma et al., 2011). It might be the possible causative intestinal bacteria in the esophageal cancer patient. However, there is an elevated threat of colon carcinoma that is interlinked with the high existence of Bacteroides vulgatus (Guarner and Malagelada, 2003).

The current study findings elaborated the significant alteration of phylum, family, genus, and species between esophageal cancer patients and control (Manichanh et al., 2008). The results indicate esophageal cancer may alter the intestinal physiology, leading to a change in the intestinal bacterial configuration. Furthermore, these variations in the intestinal bacterial composition may cause worsening of illness (Qin et al., 2010). Esophageal cancer patients' clinical signs are indigestion, vomiting, difficulty in swallowing, choking, etc. It would also be hypothesized that esophageal cancer might alter the intestinal bacterial confirmation, particularly Phylum Bacteroidetes, family Bacteroidaceae Prevotellaceae Enterobacteriaceae Veillonellaceae, genera Dialister, Prevotella_9, Escherichia-Shigella, species Bacteroides vulgatus, and Escherichia coli, also influence the whole gut bacteria. As the microbial flora influences the progression and development of esophageal carcinoma (Baba et al., 2017). Consequently, the microbiota has the potential capacity to alter the efficacy and toxicity of chemotherapy (Carmody and Turnbaugh, 2014). Current research is pretty motivating because esophageal cancer and intestinal bacteria have no direct linkage. These alterations in gut bacteria may change the host health even though illness progress is not related to the intestine (Nam et al., 2013).

Also, qPCR was performed to investigate the quantitative modification of intestinal bacteria. Results demonstrated a significant decrease of Bifidobacterium and Lactobacillus genera in esophageal cancer patients associated with preceding work (Zhou et al., 2014). Additionally, a substantial increase of Bacteroides vulgatus, and E. coli, while the insignificant rise of Clostridium leptum in the study group compared to control, is analogous to previous studies (Guarner and Malagelada, 2003; Ma et al., 2011; Wu et al., 2010).

\section{Conclusions}

In conclusion, the current study revealed significant intestinal microbiome dysbiosis between esophageal cancer patients and healthy volunteers. More specifically, there is a considerable disparity of intestinal bacterial taxa composition with the comparison of controls. The degree of certain intestinal bacteria was either showed significant increased or lowered abundance in esophageal cancer patients. The estimation of intestinal bacterial diversity shows a significantly higher level in intestinal flora of esophageal cancer than healthy volunteers, which indicates the overgrowth of gut bacteria in esophageal cancer patients. Thus, further study is required to understand the fundamental processes and mechanism of gut bacterial dysbiosis in esophageal cancer patients.

\section{Author contribution}

Hafiz Muhammad Ishaq, Jiru Xu: Study concept and design. Hafiz Muhammad Ishaq: Data acquisition. Hafiz Muhammad Ishaq, Imran Shair Mohammad: Manuscript preparation. Kiran Sher Muhammad, Huan Li, Rao Zahid Abbas, Zia ud Din Sindhu, Shakir Ullah, Yang Fan, Abbas Sadiq, Muhammad Asif Raza, Riaz Hussain, Hafiz Muhammad Arshad, Iahtasham Khan, Muhammad Umair Waqas, Aziz Ul-Rahman, Riffat Yasin, Atif Rehman, Rana Waseem Akhtar: Manuscript editing. All authors: Manuscript review.

\section{Conflict of interests}

All the authors declare no conflict of interests.

\section{Acknowledgement}

This study was supported by National Natural Science Foundation of China (No. 81730056).

\section{References}

Amani J, Ahmadpour A, Imani Fooladi AA, Nazarian S (2015). Detection of E. coli O157:H7 and Shigella dysenteriae toxins in clinical samples by PCR-ELISA. Braz J Infect Dis 19: 278-284. DOI: 10.1016/j.bjid.2015.02.008.

Atarashi K, Tanoue T, Shima T, Imaoka A, Kuwahara T, Momose Y, et al. (2011). Induction of colonic regulatory $\mathrm{T}$ cells by indigenous Clostridium species. Science 331: 337-341. DOI: 10.1126/ science.1198469.

Baba Y, Iwatsuki M, Yoshida N, Watanabe M, Baba H (2017). Review of the gut microbiome and esophageal cancer: Pathogenesis and potential clinical implications. Ann Gastroenterol Surg 1: 99-104. DOI: $10.1002 /$ ags3.12014.

Baba Y, Watanabe M, Yoshida N, Kawanaka K, Yamashita Y, Baba H (2014). Radiofrequency ablation for pulmonary metastases from gastrointestinal cancers. Ann Thor Cardiovasc Surg 20: 99-105. DOI: 10.5761/atcs.ra.13-00343.

Carmody RN, Turnbaugh PJ (2014). Host-microbial interactions in the metabolism of therapeutic and diet-derived xenobiotics. J Clin Invest 124: 4173-4181. DOI: 10.1172/JCI72335.

Chen J, Chia N, Kalari KR, Yao JZ, Novotna M, Soldan MMP, et al. (2016). Multiple sclerosis patients have a distinct gut microbiota compared to healthy controls. Scientific Reports 6: 28484. DOI: 10.1038/srep28484.

Dillon SM, Lee EJ, Kotter CV, Austin GL, Gianella S, Siewe B, et al. (2015). Gut Dendritic Cell Activation Links an Altered 
Colonic Microbiome to Mucosal and Systemic T Cell Activation in Untreated HIV-1 infection. Mucosal Immunol 9: 24-37. DOI: $10.1038 / \mathrm{mi} .2015 .33$.

Garrett WS (2015). Cancer and the microbiota. Science 348: 80-86. DOI: 10.1126/science.aaa4972.

Guarner F, Malagelada J-R (2003). Gut flora in health and disease. Lancet 361: 512-519. DOI: 10.1016/S0140-6736(03)12489-0.

Hameed A, Ijaz S, Mohammad IS, Muhammad KS, Akhtar N, Khan HMS (2017). Aglycone solanidine and solasodine derivatives: A natural approach towards cancer. Biomed Pharmacother 94: 446-457. DOI: 10.1016/j.biopha.2017.07.147.

Huang F-L, Yu S-J (2018). Esophageal cancer: Risk factors, genetic association, and treatment. Asian J Surg 41: 210-215. DOI: 10.1016/j.asjsur.2016.10.005.

Iida N, Dzutsev A, Stewart CA, Smith L, Bouladoux N, Weingarten RA, et al. (2013). Commensal bacteria control cancer response to therapy by modulating the tumor microenvironment. Science 342: 967-970. DOI: 10.1126/science.1240527.

Ishaq HM, Mohammad IS, Guo H, Shahzad M, Hou YJ, Ma C, et al. (2017a). Molecular estimation of alteration in intestinal microbial composition in Hashimoto's thyroiditis patients. Biomed Pharmacother 95: 865-874. DOI: 10.1016/j. biopha.2017.08.101.

Ishaq HM, Mohammad IS, Shahzad M, Ma C, Raza MA, Wu X, et al. (2018a). Molecular Alteration Analysis of Human Gut Microbial Composition in Graves' disease Patients. Int J Biol Sci 14: 1558-1570. DOI: 10.7150/ijbs.24151.

Ishaq HM, Shahzad M, Wu X, Ma C, Xu J (2017b). Molecular Characterization Of Fecal Microbiota Of Healthy Chinese Tobacco Smoker Subjects In Shaanxi Province, Xi'an China. J Ayub Med Coll Abbottabad 29: 3-7.

Ishaq HM, Shahzad M, Wu X, Ma C, Xu J (2018b). Gut Microbe Analysis between Asthma Patients and Healthy Volunteers in Shaanxi Province, Xi'an, China. Pakistan Journal of Zoology 50(1). DOI: 10.17582/journal.pjz/2018.50.1.165.173.

Kang D-W, Jin GP, Ilhan ZE, Wallstrom G, Labaer J, Adams JB, Krajmalnik-Brown R (2013). Reduced Incidence of Prevotella and Other Fermenters in Intestinal Microflora of Autistic Children. PloS One 8: e68322. DOI: 10.1371/journal.pone.0068322.

Kovatcheva-Datchary P, Nilsson A, Akrami R, Lee YS, De Vader F, Arora T, et al. (2015). Dietary Fiber-Induced Improvement in Glucose Metabolism Is Associated with Increased Abundance of Prevotella. Cell Metabolism 22: 971-982. DOI: 10.1016/j. cmet.2015.10.001.

Ma C, Wu X, Nawaz M, Li J, Yu P, Moore JE, Xu J (2011). Molecular characterization of fecal microbiota in patients with viral diarrhea. Curr Microbiol 63: 259-266. DOI: 10.1007/s00284-011-9972-7.

Manichanh C, Varela E, Martinez C, Antolin M, Llopis M, Doré J, et al. (2008). The gut microbiota predispose to the pathophysiology of acute postradiotherapy diarrhea. Am J Gastroenterol 103: 1754-1761. DOI: 10.1111/j.1572-0241.2008.01868.x.

Mao A (2016). Interventional Therapy of Esophageal Cancer. Gastrointest Tumors 3: 59-68. DOI: 10.1159/000447512.

Mohammad IS, Chaurasiya B, Yang X, Lin C, Rong H, He W (2020a). Homotype-Targeted Biogenic Nanoparticles to Kill MultidrugResistant Cancer Cells. Pharmaceutics 12: 950. DOI: 10.3390/ pharmaceutics12100950.

Mohammad IS, He W, Yin L (2018a). A smart paclitaxel-disulfiram nanococrystals for efficient MDR reversal and enhanced apoptosis. Pharm Res 35: 77. DOI: 10.1007/s11095-018-2370-0.

Mohammad IS, He W, Yin L (2018b). Understanding of human ATP binding cassette superfamily and novel multidrug resistance modulators to overcome MDR. Biomed Pharmacother 100: 335-348. DOI: 10.1016/j.biopha.2018.02.038.
Mohammad IS, He W, Yin L (2020b). Insight on Multidrug Resistance and Nanomedicine Approaches to Overcome MDR. Crit Rev Ther Drug Carrier Syst 37: 473-509. DOI: 10.1615/ CritRevTherDrugCarrierSyst.2020025052.

Mulvey MA, Schilling JD, Martinez JJ, Hultgren SJ (2000). Bad bugs and beleaguered bladders: Interplay between uropathogenic Escherichia coli and innate host defenses. Proc Natl Acad Sci U S A 97: 8829-8835. DOI: 10.1073/pnas.97.16.8829.

Nam Y-D, Kim HJ, Seo J-G, Kang SW, Bae J-W (2013). Impact of Pelvic Radiotherapy on Gut Microbiota of Gynecological Cancer Patients Revealed by Massive Pyrosequencing. PloS One 8: e82659. DOI: 10.1371/journal.pone.0082659.

Pittayanon R, Lau JT, Yuan Y, Leontiadis GI, Tse F, Surette M, Moayyedi P (2019). Gut microbiota in patients with irritable bowel syndrome - a systematic review. Gastroenterology 157: 97-108. DOI: 10.1053/j.gastro.2019.03.049.

Power SE, O'Toole PW, Stanton C, Ross RP, Fitzgerald GF (2013). Intestinal microbiota, diet and health. Br J Nutr 111: 387-402. DOI: 10.1017/S0007114513002560.

Qin J, Li R, Raes J, Arumugam M, Burgdorf KS, Manichanh C, et al. (2010). A human gut microbial gene catalogue established by metagenomic sequencing. Nature 464: 59-65. DOI: 10.1038/ nature08821.

Strati F, Cavalieri D, Albanese D, De Felice C, Donati C, Hayek J, et al. (2017). New evidences on the altered gut microbiota in autism spectrum disorders. Microbiome 5: 24. DOI: 10.1186/ s40168-017-0242-1.

The Cancer Genome Atlas Research Network (2017). Integrated genomic characterization of oesophageal carcinoma. Nature 541: 169-175. DOI: $10.1038 /$ nature20805.

Triboulet J-P, Mariette C (2008). Oesophageal cancer: what's new during the last 10 years? Bulletin du Cancer 95: 425-431. DOI: $10.1684 / \mathrm{bdc} .2008 .0626$.

Van Der Gucht K, Sabbe K, De Meester L, Vloemans N, Zwart G, Gillis M, Vyverman W (2001). Contrasting bacterioplankton community composition and seasonal dynamics in two neighbouring hypertrophic freshwater lakes. Environ Microbiol 3: 680-690. DOI: 10.1046/j.1462-2920.2001.00242.x.

Walsh CJ, Guinane CM, O'Toole PW, Cotter PD (2014). Beneficial modulation of the gut microbiota. FEBS Lett 588: 4120-4130. DOI: 10.1016/j.febslet.2014.03.035.

Walters WA, Xu Z, Knight R (2014). Meta-analyses of human gut microbes associated with obesity and IBD. FEBS Lett 588: 4223-4233. DOI: 10.1016/j.febslet.2014.09.039.

Wang Y, Luo X, Mao X, Tao Y, Ran X, Zhao H, Xiong J, Li L (2017). Gut microbiome analysis of type 2 diabetic patients from the Chinese minority ethnic groups the Uygurs and Kazaks. PLoS One 12: e0172774. DOI: 10.1371/journal.pone.0172774.

Wu X, Ma C, Han L, Nawaz M, Gao F, Zhang X, et al. (2010). Molecular characterization of the faecal microbiota in patients with type II diabetes. Curr Microbiol 61: 69-78. DOI: 10.1007/ s00284-010-9582-9.

Zaidi AH, Kelly LA, Kreft RE, Barlek M, Omstead AN, Matsui D, et al. (2016). Associations of microbiota and toll-like receptor signaling pathway in esophageal adenocarcinoma. BMC Cancer 16: 52. DOI: 10.1186/s12885-016-2093-8.

Zhang M, Zheng M, Wu Z, Guan M, Liu S, Zhao W, Cheng J (2017). Alteration of the gut microbial community after N,NDimethylformamide exposure. J Toxicol Sci 42: 241-250. DOI: $10.2131 /$ jts.42.241.

Zhou L, Li X, Ahmed A, Wu D, Liu L, Qiu J, et al. (2014). Gut microbe analysis between hyperthyroid and healthy individuals. Curr Microbiol 69: 675-680. DOI: 10.1007/s00284-014-0640-6. 


\section{Supplementary material}

Table 1. The type and grade of esophageal cancer patients recruited

\begin{tabular}{ccccc}
\hline Patient & Age & Sex & Type of carcinoma & Cancer grade \\
\hline 1 & 55 & Male & Squamous cell carcinoma & Grade 2 \\
2 & 60 & Male & Squamous cell carcinoma & Grade 3 \\
3 & 45 & Male & Adenocarcinoma 3 \\
4 & 53 & Male & Squamous cell carcinoma & Grade 2 \\
5 & 55 & Male & Adenocarcinoma & Grade 2 \\
6 & 51 & Male & Squamous cell carcinoma & Grade 2 \\
7 & 56 & Male & Squamous cell carcinoma & Grade 2 \\
8 & 55 & Male & Squamous cell carcinoma & Grade 2 \\
9 & 49 & Male & Squamous cell carcinoma & Grade 3 \\
11 & 52 & Male & Adenocarcinoma & Grade 2 \\
12 & 60 & Female & Squamous cell carcinoma & Grade 2 \\
13 & 52 & Female & Squamous cell carcinoma & Grade 3 \\
14 & 57 & Female & Squamous cell carcinoma & Grade 2 \\
\hline
\end{tabular}

Table 2. Linkage Primers deployed in PCR-DGGE and qPCR

\begin{tabular}{|c|c|c|}
\hline Target bacteria & & Primer Sequence (5'-3') \\
\hline \multicolumn{3}{|c|}{ PCR-DGGE Primer } \\
\hline $341-\mathrm{F}$ & & CCTACGGGAGGCAGCAG \\
\hline $534-\mathrm{R}$ & & ATT ACCGCGGCTGCTGG \\
\hline \multirow[t]{2}{*}{$341 \mathrm{FG}$} & & CGCCCGCCGCGCGCGGCGGCGCGGGGCGGGGGCACGGGGGGCCTACGGGAGGCAGCAG \\
\hline & \multicolumn{2}{|c|}{ Real-Time PCR Primer } \\
\hline \multirow{2}{*}{$\begin{array}{l}\text { Bifidobacterium } \\
(550 \mathrm{bp})\end{array}$} & Bifid F & CTC CTGGAAACGGGTGG \\
\hline & Bifi-R & GGTGTTCTTCCCGATATCTACA \\
\hline \multirow{2}{*}{$\begin{array}{l}\text { Lactobacillus } \\
(250 \mathrm{bp})\end{array}$} & Lact F & CTCAAAACTAAACAAAGTTTC \\
\hline & Lact $\mathrm{R}$ & CTCAAAACT AAACAAAGTTTC \\
\hline \multirow{2}{*}{$\begin{array}{l}\text { Bacteroides vulgatus } \\
(287 \mathrm{bp})\end{array}$} & BV-F & GCATCATGAGTCCGCATGTTC \\
\hline & BV-R & TCCATACCCGACTTTATTCCTT \\
\hline \multirow{2}{*}{$\begin{array}{l}\text { Escherichia coli } \\
(287 \mathrm{bp})\end{array}$} & E. coli-F & CATTGACGTTACCGCAGAAGAAGC \\
\hline & E. coli-R & CTCTACGAGACTCAAGCTTGC \\
\hline \multirow{2}{*}{$\begin{array}{l}\text { Clostridium leptum } \\
(239 \mathrm{bp})\end{array}$} & C. lep-F & GCACAAGCAGTGGAGT \\
\hline & C. lep-R & CTTCCTCCGTTTTGTCAA \\
\hline
\end{tabular}

Table 3. Gut microbial diversity and similarity of esophageal cancer patients and healthy control

\begin{tabular}{lcccc}
\hline Groups & \multicolumn{2}{c}{ Diversity } & \multicolumn{2}{c}{ Similarity } \\
\cline { 2 - 5 } & Number of bands $^{\mathrm{a}}$ & Shannon index & Intra-similarity $^{\mathrm{c}}$ & Inter-similarity $^{\mathrm{d}}$ \\
\hline Patients group & $12.12 \pm 3.37$ & $3.06 \pm 0.69$ & $0.340 \pm 0.164$ & $0.174 \pm 0.155$ \\
Healthy control & $7.73 \pm 1.60$ & $2.52 \pm 0.26$ & $0.271 \pm 0.147$ & $/$ \\
$P$-value & 0.0007 & 0.017 & $/$ & $/$ \\
\hline
\end{tabular}

Significantly different through, unpaired $t$ test, with $(P<0.05)$.

${ }^{a}$ DGGE bands number produced by each sample; ${ }^{b}$ Shannon-Weaver diversity index $\left(H^{1}\right)$ was evaluated in each sample by using (relative) intensities of all DGGE bands; ${ }^{c}$ Comparing of DGGE band profiles with Dice similarity coefficients within the individual of a given group; ${ }^{\mathrm{d}}$ Comparing of DGGE band profiles by using Dice similarity coefficients between members of esophageal cancer patients and healthy group. 
Table 4. Sequencing of excised PCR-DGGE bands and identification by using the BLAST database

\begin{tabular}{|c|c|c|c|c|}
\hline $\begin{array}{l}\text { Selected exised } \\
\text { bands }\end{array}$ & Bacteria with highest $\%$ homology & $\begin{array}{l}\text { Sequence accession } \\
\text { number }\end{array}$ & Bacterial phyla & Gene bank number \\
\hline D1a & Escherichia coli (98) & O104:H4 & Proteobacteria & NC_018658.1 \\
\hline D2a & Bacteroides fluxus (89) & YIT 12057 & Bacteroidetes & NZ_GL882626.1 \\
\hline D3a & Enterobacter sacchari (96) & SP1 & Firmicutis & NZ_CP007215.2 \\
\hline D3b & Prevotella copri (96) & DSM 18205 & Bacteroidetes & NZ_GG703856.1 \\
\hline $\mathrm{D} 4 \mathrm{a}$ & Shigella flexneri (98) & 2a str. 301 & Proteobacteria & NC_004337.2 \\
\hline $\mathrm{D} 4 \mathrm{~b}$ & Bacillus cecembensis (98) & DSM 21993 & Firmicutis & NZ_LMBZ01000203.1 \\
\hline D5a & Proteus mirabilis (94) & HI4320 & Firmicutis & NC_010554.1 \\
\hline D6a & Bacteroides vulgatus (97) & ATCC 8482 & Bacteroidetes & NC_009614.1 \\
\hline D7a & Bacteroides uniformis (95) & CL03T00C23 & Bacteroidetes & NZ_JH724260.1 \\
\hline D8a & Bacteroides salyersiae (94) & DSM 18765 & Bacteroidetes & NZ_KB905470.1 \\
\hline $\mathrm{C} 1 \mathrm{a}$ & Bacteroides stercoris (94) & ATCC 43183 & Bacteroidetes & NZ_DS499676.1 \\
\hline C3a & Prevotella copri (96) & DSM 18205 & Bacteroidetes & NZ_GG703856.1 \\
\hline C4a & Bacteroides coprocola (97) & DSM 17136 & Bacteroidetes & NZ_DS981495.1 \\
\hline D9a & Bacteroides plebeius (91) & DSM 17135 & Bacteroidetes & NZ_DS990123.1 \\
\hline D9b & Bacteroides oleiciplenus (91) & YIT 12058 & Bacteroidetes & NZ_JH992946.1 \\
\hline D13a & Klebsiella pneumonia (93) & HS11286 & Firmicutes & NC_016845.1 \\
\hline D14a & Klebsiella pneumonia (93) & HS11286 & Firmicutes & NC_016845.1 \\
\hline D15a & Bacillus sp. (99) & FJAT-25496 & Firmicutes & NZ_LMBY01000086.1 \\
\hline D15b & Pseudomonas cremoricolorata (98) & ND07 & Proteobacteria & NZ_CP009455.1 \\
\hline $\mathrm{D} 15 \mathrm{C}$ & Enterobacter aerogenes (92) & КСТС 2190 & Proteobacteria & NC_015663.1 \\
\hline D15b & Megasphaera sp. (94) & DJF_B143 & Firmicutis & NZ_LODR01000015.1 \\
\hline $\mathrm{C} 6 \mathrm{a}$ & Bacteroides dorei (92) & HS2L2B045b & Firmicutis & СР009057.1 \\
\hline $\mathrm{C} 10 \mathrm{a}$ & Bacteroides caecimuris (92) & I48 & Bacteroidetes & NZ_CP015401.1 \\
\hline
\end{tabular}

Table 5. Different bacterial quantification outcomes by qPCR

\begin{tabular}{lccc}
\hline Bacteria & Control & Patients & $P$-value \\
\hline Bifidobacterium $\left(10^{5}\right)$ & $8.07 \pm 1.04$ & $6.91 \pm 1.08$ & 0.0068 \\
Bacteroides vulgatus $\left(10^{7}\right)$ & $6.92 \pm 1.03$ & $7.93 \pm 1.02$ & 0.0119 \\
Lactobacillus $\left(10^{4}\right)$ & $7.98 \pm 0.89$ & $7.12 \pm 1.04$ & 0.0221 \\
Escherichia coli & $3.90 \pm 0.85$ & $4.59 \pm 0.78$ & 0.0241 \\
Clostridium leptum $\left(10^{5}\right)$ & $4.12 \pm 1.22$ & $4.39 \pm 1.18$ & 0.2928 \\
\hline
\end{tabular}

Data presented as the average estimate of logarithms of fecal PCR target genetic amplicon, copy numbers present in $1 \mathrm{~g}$ of feces.

Table 6. High-throughput intestinal bacterial diversity index based on the $97 \%$ similarity analysis

\begin{tabular}{lcccccccc}
\hline Group & $\begin{array}{c}\text { Observed } \\
\text { species }\end{array}$ & OTUs & Shannon & Simpson & Chao1 & ACE & $\begin{array}{c}\text { Good' } \\
\text { coverage }\end{array}$ \\
\hline Patients & 278.6 & 288.1 & 5.05 & 0.943 & 289.23 & 295.59 & 0.9997 & 0.387 \\
Control & 253.8 & 259.5 & 4.35 & 0.909 & 257.00 & 268.03 & 0.9994 & 0.334 \\
$P$-value & 0.0174 & 0.0414 & 0.0226 & 0.0222 & 0.0170 & 0.0348 & 0.0982 & 0.0237 \\
\hline
\end{tabular}

The mean values of each group shown in the table, unpaired $t$-test $t$ were used to signify the results, where $(P<0.05)$. The evenness based on Shannon, calculated by using formula $\mathrm{E}=\mathrm{H} / \mathrm{ln}(\mathrm{S})$; where $\mathrm{H}$ denotes Shannon index of diversity and $\mathrm{S}$ represents whole number sequences in a particular group. 
Table 7. Esophageal cancer intestinal bacterial phylotypes obtained from High-throughput sequencing

\begin{tabular}{|c|c|c|c|c|c|c|}
\hline Taxa & Mean D & Mean C & $P$-value & Q-value & $\% \mathrm{D}$ & $\% C$ \\
\hline \multicolumn{7}{|l|}{ Phylum } \\
\hline Firmicutes & 0.33616 & 0.44690 & 0.15313 & 0.74408 & 33.62 & 44.69 \\
\hline Bacteroidetes & 0.58414 & 0.34977 & 0.03143 & 0.63178 & 58.41 & 34.98 \\
\hline Proteobacteria & 0.07261 & 0.15099 & 0.08494 & 0.63315 & 7.26 & 15.10 \\
\hline Verrucomicrobia & 0.00108 & 0.01061 & 0.67300 & 0.96243 & 0.11 & 1.06 \\
\hline Tenericutes & 0.00165 & 0.01003 & 0.32144 & 0.68118 & 0.17 & 1.00 \\
\hline Cyanobacteria & 0.00020 & 0.01007 & 0.33450 & 0.76168 & 0.02 & 1.01 \\
\hline Actinobacteria & 0.00319 & 0.01047 & 0.15139 & 0.76178 & 0.32 & 1.05 \\
\hline Fusobacteria & 0.00069 & 0.00115 & 0.53111 & 0.71220 & 0.07 & 0.11 \\
\hline Acidobacteria & 0.00011 & 0.00881 & 0.10569 & 0.74038 & 0.01 & 0.88 \\
\hline Synergistetes & 0.00001 & 0.00104 & 0.27426 & 0.69438 & 0.00 & 0.10 \\
\hline Others & & & & & 0.02 & 0.01 \\
\hline \multicolumn{7}{|l|}{ Family } \\
\hline Bacteroidaceae & 0.35432 & 0.17513 & 0.03211 & 0.73533 & 35.43 & 17.51 \\
\hline Ruminococcaceae & 0.14991 & 0.20079 & 0.22533 & 0.70320 & 14.99 & 20.08 \\
\hline Prevotellaceae & 0.03579 & 0.19696 & 0.02641 & 0.64459 & 3.58 & 19.70 \\
\hline Lachnospiraceae & 0.09669 & 0.09224 & 0.43025 & 0.75133 & 9.67 & 9.22 \\
\hline Enterobacteriaceae & 0.06367 & 0.02065 & 0.04321 & 0.69323 & 6.37 & 2.07 \\
\hline Alcaligenaceae & 0.05654 & 0.06651 & 0.42342 & 0.86213 & 5.65 & 6.65 \\
\hline Veillonellaceae & 0.02649 & 0.06263 & 0.03751 & 0.61437 & 2.65 & 6.26 \\
\hline Porphyromonadaceae & 0.06416 & 0.05181 & 0.71409 & 0.89288 & 6.42 & 5.18 \\
\hline Rikenellaceae & 0.06186 & 0.05754 & 0.64235 & 0.95458 & 6.19 & 5.75 \\
\hline unidentified_Chloroplast & 0.02112 & 0.01011 & 0.82317 & 0.84133 & 2.11 & 1.01 \\
\hline Others & & & & & 6.95 & 6.56 \\
\hline \multicolumn{7}{|l|}{ Genus } \\
\hline Bacteroides & 0.30432 & 0.20513 & 0.04361 & 0.51558 & 30.43 & 20.51 \\
\hline Prevotella_9 & 0.03349 & 0.18523 & 0.02183 & 0.61072 & 3.35 & 18.52 \\
\hline Alistipes & 0.06186 & 0.05754 & 0.71837 & 0.86348 & 6.19 & 5.75 \\
\hline Escherichia-Shigella & 0.05052 & 0.00155 & 0.03540 & 0.63280 & 5.05 & 0.16 \\
\hline Ruminococcus_2 & 0.02725 & 0.03395 & 0.81108 & 0.88648 & 2.73 & 3.40 \\
\hline Dialister & 0.00813 & 0.04356 & 0.03677 & 0.62039 & 0.81 & 4.36 \\
\hline Parasutterella & 0.05810 & 0.02785 & 0.13753 & 0.61528 & 5.81 & 2.78 \\
\hline Faecalibacterium & 0.04549 & 0.02653 & 0.11030 & 0.72153 & 4.55 & 2.65 \\
\hline Parabacteroides & 0.03649 & 0.03189 & 0.51916 & 0.87121 & 3.65 & 3.19 \\
\hline $\begin{array}{l}\text { [Eubacterium]_ } \\
\text { coprostanoligenes_group }\end{array}$ & 0.02417 & 0.03251 & 0.57431 & 0.92243 & 2.42 & 3.25 \\
\hline Others & & & & & 35.02 & 35.43 \\
\hline
\end{tabular}

Significantly dissimilar $P<0.05, \mathrm{D}$ and $C$ represent esophageal cancer and control. 
Table 8. Species levels intestinal bacterial richness by High-throughput outcomes

\begin{tabular}{|c|c|c|c|c|}
\hline Taxa & Mean D & Mean C & $P$-value & Q-value \\
\hline Bacteroides vulgatus & 0.211467 & 0.032123 & 0.03461 & 0.62015 \\
\hline Escherichia coli & 0.071835 & 0.011431 & 0.03262 & 0.64025 \\
\hline Bacteroides uniformis & 0.065836 & 0.023431 & 0.06444 & 0.74025 \\
\hline Bacteroides plebeius & 0.026473 & 0.015168 & 0.20426 & 0.86106 \\
\hline Bacteroides stercoris & 0.033955 & 0.021475 & 0.0464 & 0.73016 \\
\hline Bacteroides coprophilus & 0.015334 & 0.005112 & 0.0753 & 0.72186 \\
\hline Bacteroides massiliensis & 0.012785 & 0.004763 & 0.4156 & 0.87008 \\
\hline Parabacteroides distasonis & 0.031134 & 0.014976 & 0.31257 & 0.83664 \\
\hline Haemophilus parainfluenzae & 0.015944 & 0.008915 & 0.32418 & 0.89188 \\
\hline Sutterella wadsworthensis & 0.021251 & 0.001865 & 0.13455 & 0.70193 \\
\hline Fritillaria taipaiensis & 0.022116 & 0.000211 & 0.13104 & 0.99178 \\
\hline Lactobacillus casei & 0.001267 & 0.005583 & 0.03174 & 0.71172 \\
\hline [Clostridium] leptum & 0.012471 & 0.007118 & 0.13366 & 0.71766 \\
\hline Paraprevotella clara & 0.00056 & 0.004652 & 0.02796 & 0.28954 \\
\hline Ruminococcus bicirculans & 0.004221 & 0.006382 & 0.56024 & 0.87372 \\
\hline Bacteroides fragilis & 0.009088 & 0.003979 & 0.15782 & 0.67786 \\
\hline salivarius subsp. thermophilus & 0.008903 & 0.000342 & 0.1118 & 0.5862 \\
\hline Bifidobacterium longum & 0.000766 & 0.004095 & 0.16671 & 0.74025 \\
\hline Bacteroides ovatus & 0.03718 & 0.01544 & 0.07581 & 0.52122 \\
\hline Bifidobacterium kashiwanohense & 0.001315 & 0.005971 & 0.1049 & 0.4951 \\
\hline Shigella flexneri & 0.013466 & 0.012497 & 0.57631 & 0.88965 \\
\hline Bacteroides dorei & 0.003696 & 0.000327 & 0.27551 & 0.82114 \\
\hline Paraprevotella clara & 0.00054 & 0.004562 & 0.02885 & 0.28754 \\
\hline Phascolarctobacterium faecium & 0.007023 & 0.002458 & 0.16751 & 0.84117 \\
\hline Dialister propionicifaciens & $6.58 \mathrm{E}-04$ & $1.05 \mathrm{E}-04$ & 0.01896 & 0.34236 \\
\hline Megasphaera micronuciformis & 0.000212 & $7.18 \mathrm{E}-04$ & 0.03894 & 0.43561 \\
\hline Ruminococcus sp._5_1_39BFAA & 0.002698 & 0.001381 & 0.21856 & 0.82117 \\
\hline Alistipes shahii & 0.01263 & 0.009748 & 0.4023 & 0.88107 \\
\hline Alistipes indistinctus & 0.000718 & 0.002913 & 0.35365 & 0.85024 \\
\hline
\end{tabular}

